\title{
Moockinho: uma plataforma aberta de cursos online, gratuitos e massivos
}

\author{
Pedrina C. Brasil ${ }^{1}$, Andreíza de P. da S. Eleuterio ${ }^{1}$, Robson E. F. da Silva ${ }^{1}$ \\ ${ }^{1}$ Coordenação de Informática - Instituto Federal de Educação, Ciência e Tecnologia do \\ Rio Grande do Norte - Campus Avançado de Lajes (IFRN) \\ Rodovia BR 304, Km 120 - Centro - Lajes-RN \\ pedrina.brasileifrn.edu.br, \{andreizalajes, rossbaldini20\}@gmail.com
}

\begin{abstract}
Resumo. Atualmente é crescente a tendência de inserção de métodos, técnicas e tecnologias de Educação a Distância (EaD) em um sistema integrado de ensino que permita a combinação de recursos de aprendizagem que podem ser utilizados em cursos presenciais elou totalmente a distância. Os Massively Open Online Courses (MOOC) é um modelo de cursos, com conteúdo distribuído de forma livre, aberta e acessivel a qualquer pessoa de qualquer lugar, por meio da internet. Embora a largamente utilizadas, essas plataformas em geral são proprietárias e restringem as funcionalidades de seus usuários. Nesse contexto, este trabalho como objetivo apresentar uma plataforma de código aberto (open source), que permita seus usuários atender, divulgação e gerenciar de cursos.
\end{abstract}

\section{Cenário de uso}

Por meio da Internet, pessoas compartilham o conhecimento que antes era restrito a um pequeno grupo de indivíduos. A Internet, como meio de comunicação e colaboração, tem o potencial de conectar as pessoas, colocando-as em um modo de produção colaborativo (DA MATTA e FIGUEIREDO, 2013).

Em consonância com crescimento da área educacional e o desenvolvimento de novos recursos tecnológicos de informação e comunicação, surge o conceito de Massive Open Online Courses (MOOC) - em português Cursos Online Abertos e Massivos. MOOC pode ser definido como um modelo de curso que integra três elementos básicos: a conectividade das redes sociais, o conhecimento de especialistas de negócio (professores/instrutores) e a coleção de recursos educativos online passíveis de serem acessados por qualquer pessoa, em qualquer lugar. Não só estes elementos são importantes, mas também o número de inscritos em um curso. Neste sentido, sua principal característica, reside no fato deste modelo construir um engajamento ativo de dezenas ou centenas de milhares de estudantes que se auto organizam para atingir suas metas (CREED-DIKEOGU; CLARK,2013).

Visando tirar vantagem do atual contexto e ampliar o acesso da sociedade aos recursos educacionais produzidos/utilizados nas instituições de ensino, públicas ou privadas, de nível fundamental, básico e superior é proposta a plataforma Moockinho. A plataforma Moockinho é um sistema web que possibilita a educadores disponibilizar recursos educacionais através de cursos online, abertos e massivos. Através dela, educadores podem, por exemplo, gravar suas aulas (no formato de videoaula) e indexálas virtualmente, possibilitando que os demais usuários possam acessar esse recurso de qualquer lugar, a qualquer momento. Alunos, por sua vez, podem, por exemplo, 
VII Congresso Brasileiro de Informática na Educação (CBIE 2018)

Anais dos Workshops do VII Congresso Brasileiro de Informática na Educação (WCBIE 2018)

engajar-se nos cursos da plataforma, assistir suas videoaulas e acompanhar seu progresso de aprendizagem.

O nome Moockinho foi inspirado na sigla MOOC. Plataformas MOOC além de oferecer videoaulas, leituras complementares, testes e referências convidam os alunos a interagir em fóruns para discutir e sanar dúvidas. Entre as plataformas MOOC mais utilizadas no Brasil está a plataforma TimTec. A TimTec ${ }^{1}$ foi a primeira plataforma MOOC desenvolvida inteiramente no Brasil. Criada em 2013 com o objetivo de disponibilizar cursos online, livres e gratuitos voltados à educação profissional e tecnológica. Essa é um software livre que pode ser instalado por qualquer instituição de ensino ou organização interessada em ter sua própria plataforma MOOC. Qualquer usuário pode atender as aulas e obter um certificado de horas ao final do curso. Institutos federais, universidades, organizações do sistema de educação e outras instituições de ensino têm usado a plataforma para potencializar as ações de educação a distância da Rede e-Tec Brasil (INSTITUTO TIM, 2018). Entretanto, para utilizar os recursos administrativos da plataforma, por exemplo: adicionar cursos/recursos de aprendizagem, é preciso instalar a plataforma em um servidor de aplicação próprio.

O Moockinho é um software livre que visa facilitar a indexação e promoção de cursos online de forma que qualquer educador possa acessar a plataforma (http://lausana.ifrn.edu.br/moockinho), cadastrar novos cursos e videoaulas sem necessariamente instalar o sistema. Além disso, qualquer usuário pode acessar a plataforma (http://lausana.ifrn.edu.br/moockinho), se cadastrar, engajar-se nos cursos disponíveis, assistir suas videoaulas e acompanhar seu progresso de aprendizagem.

\section{Desenvolvimento}

O processo de desenvolvimento utilizado neste projeto foi baseado na metodologia scrum. Essa foi utilizada como base para acompanhamento e planejamento das atividades desenvolvimento. Esse foi dividido em seis ciclos de execução, também denominados de sprints, conforme ilustrado no Tabela 1

Tabela 1. Sprints do Projeto

\begin{tabular}{|c|c|}
\hline Sprint & Atividade \\
\hline 1 & Levantamento e Elaboração de Requisitos \\
\hline 2 & Análise e projeto do Sistema \\
\hline 3 & Implementação dos casos de uso de maior risco \\
\hline 4 & Implementação dos casos de uso secundários \\
\hline 5 & Testes Unitários e de Integração \\
\hline 6 & Implantação no servidor de aplicação e Validação com Usuários \\
\hline
\end{tabular}

${ }^{1}$ http://timtec.com.br/pt/ 
Cada sprint teve duração média de 20 (vinte) a 30 (trinta) dias consecutivos de execução. E, ao final de cada sprint, era realizada uma reunião onde os resultados da equipe e os aprendizados daquele ciclo eram compartilhados. Em seguida a equipe selecionava as atividades a serem realizadas no próximo ciclo de execução.

Ao total 6 sprints foram realizadas para atender as atividades de iniciação, elaboração, construção e transição deste trabalho.

Para construção do sistema proposto foram pesquisadas tecnologias que facilitassem o desenvolvimento de sistemas web. Entre as tecnologias encontradas foram selecionados o Play Framework e o Twitter Bootstrap devido sua flexibilidade e facilidade de aprendizagem.

Paralelo a elas foram também utilizados: o ambiente de desenvolvimento integrado Netbeans para edição de código; o banco de dados MySQL Server, para armazenamento de dados; a linguagem de programação J2EE, para implementação da interface servidora deste projeto; e HTML, CSS e Javascript para implementação da interface cliente do sistema projetado.

A plataforma foi implantada num servidor de aplicação do Instituto Federal de Educação, Ciência e Tecnologia do Rio Grande do Norte (IFRN) e está atualmente em fase de validação. Nesse os servidores e alunos do IFRN estão criando e compartilhando cursos com o objetivo de validar a proposta do sistema. Passada a etapa de validação, a plataforma Moockinho será promovida e disponibilizada para implantação nas demais instituições de ensino da Rede e-TEC e secretarias de educação. O Moockinho é a primeira plataforma MOOC desenvolvida inteiramente no Nordeste.

\section{Apresentação do Software}

O Moockinho é um software educacional com objetivo de disponibilizar cursos online, livres e gratuitos. Através desse, os usuários poderão se inscrever nos cursos e ter acesso às videoaulas cadastradas no sistema. Este é dividido em 3 módulos: Módulo Público; Módulo de Aluno; e Módulo de Administrador.

O módulo público é utilizado pelos usuários avulsos que não estão matriculados no sistema, chamados aqui de usuários avulsos, esses poderão visualizar cursos, cadastrar-se no sistema, detalhar cursos, efetuar login e recuperar senha. 
VII Congresso Brasileiro de Informática na Educação (CBIE 2018)

Anais dos Workshops do VII Congresso Brasileiro de Informática na Educação (WCBIE 2018)

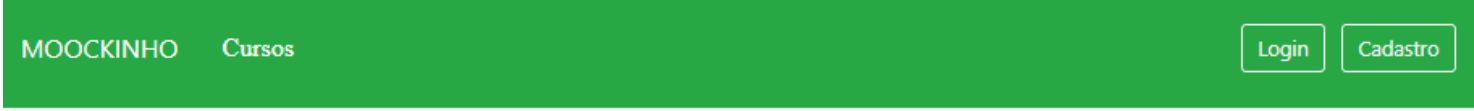

Todos os Cursos

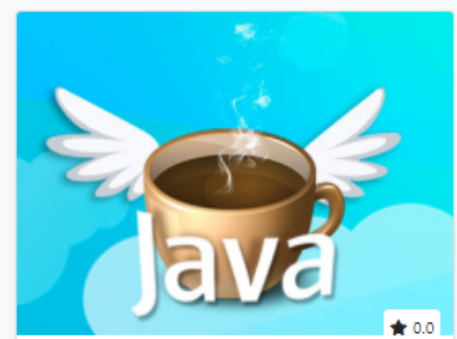

Curso de Java

Inicio: 2018-07-10 | Prof: administrador

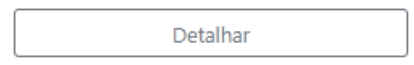

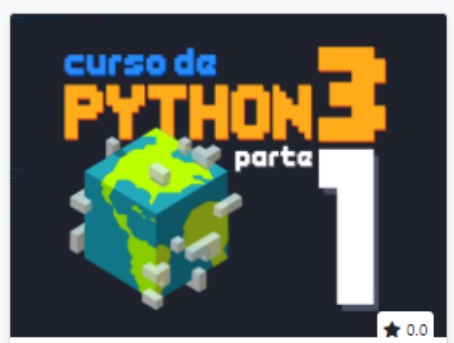

Curso de Python 3

Início: 2018-07-13 | Prof: administrador

Detalhar

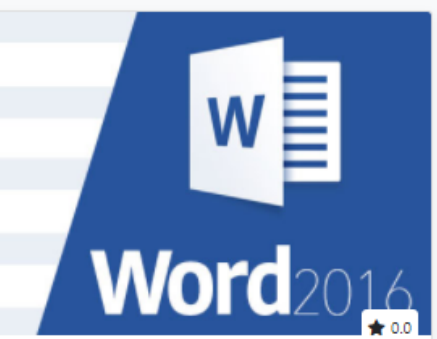

Curso de Word 2016

Inicio: 2018-07-17 | Prof:: administrador

Figura 1a. Visualizar todos os Cursos

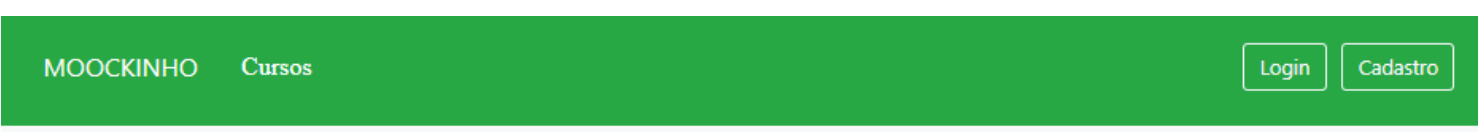

\section{Curso de Java}

Data de Inicio: 2018-07-10 | Prof: aaministrador | Avaliação: 0.0

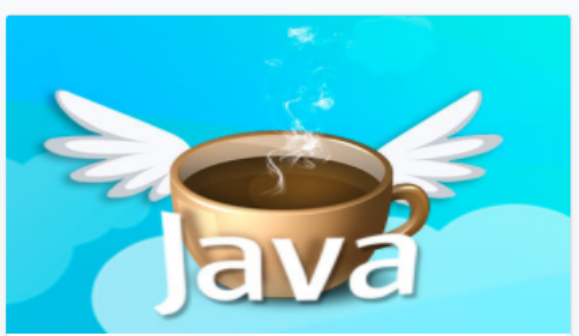

O Java é a Linguagem de Programação mais famosa do mundo. Atualmente, encontramos aplicativos Java rodando em computadores, smartphones, tablets, video-games e até mesmo em cartões de crédito e sondas espaciais da NASA. Nesse curso de Java grátis e completo, o

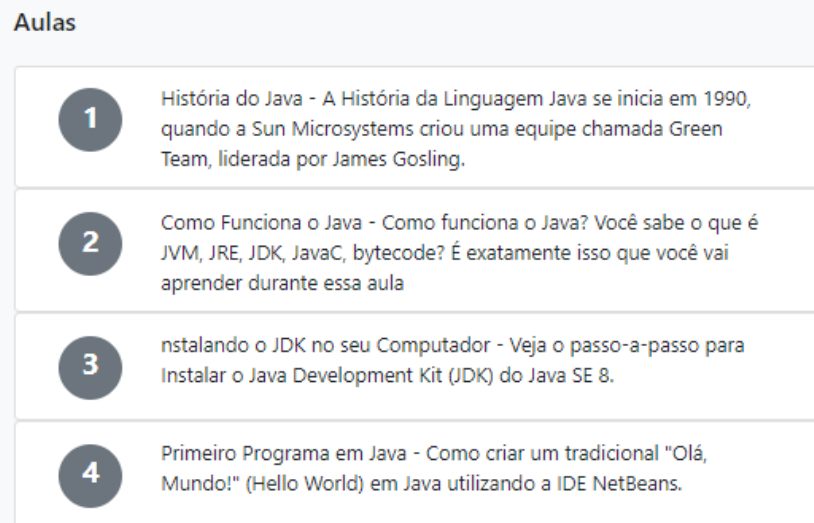

Aulas

Figura 1b. Detalhar Curso

O módulo aluno é utilizado pelos usuários com registro no sistema. Além de fazer tudo que um usuário avulso faz, podem editar perfil de cadastro, realizar matrícula em cursos do sistema, cancelar matrícula, acompanhar progresso de aprendizagem, assistir videoaulas e avaliar cursos que estiver matriculado, realizar comentários a videoaulas e solicitar promoção a administrador. 
VII Congresso Brasileiro de Informática na Educação (CBIE 2018)

Anais dos Workshops do VII Congresso Brasileiro de Informática na Educação (WCBIE 2018)

S Meu Perfil Minhas Matriculas U Sair

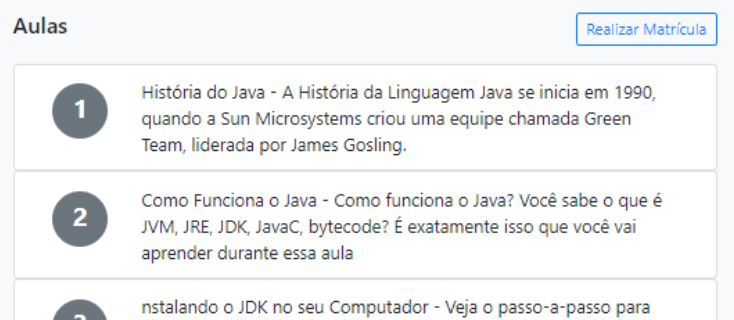

Figura 2a. Realizar Matrícula professor Gustavo Guanabara vai mostrar como criar aplicativos que utilizem Java para rodar em Desktop. Será usado Java SE 8 com IDE NetBeans e os exemplos serão feitos usando a API Swing, que cria interfaces gráficas atraentes.

Registre sua avaliaçào:

(1) $2 \circ 3 \circ 5 \circ$

Salvar Avaliação

\section{Curso de Java}

Aula 1 - História do Java

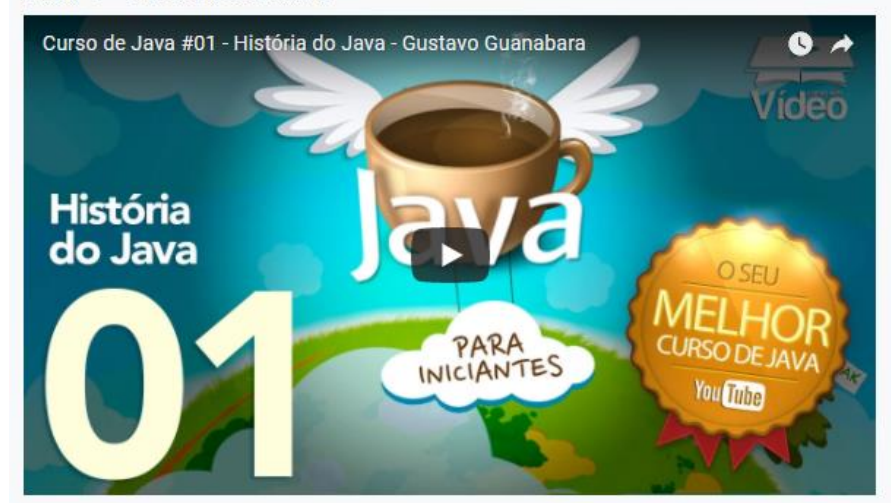

Figura 2b. Avaliar Curso

Novo Comentário

Figura 2c. Assistir Videoaula

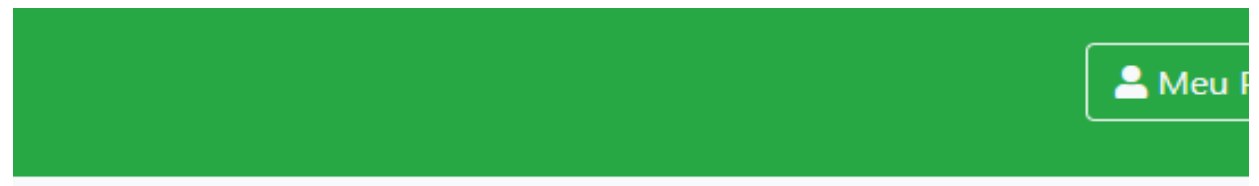

\section{Solicitar Promoção}

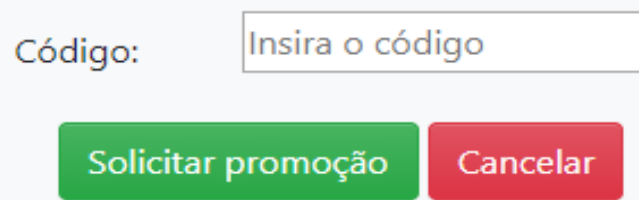

Figura 2d. Solicitar Promoção a Administrador 
VII Congresso Brasileiro de Informática na Educação (CBIE 2018)

Anais dos Workshops do VII Congresso Brasileiro de Informática na Educação (WCBIE 2018)

O módulo de administrador é utilizado pelos usuários com registro no sistema e privilégios administrativos. Uma vez solicitada a promoção, um usuário, além de fazer tudo que um usuário registrado faz, pode gerenciar cursos e gerenciar videoaulas no sistema.

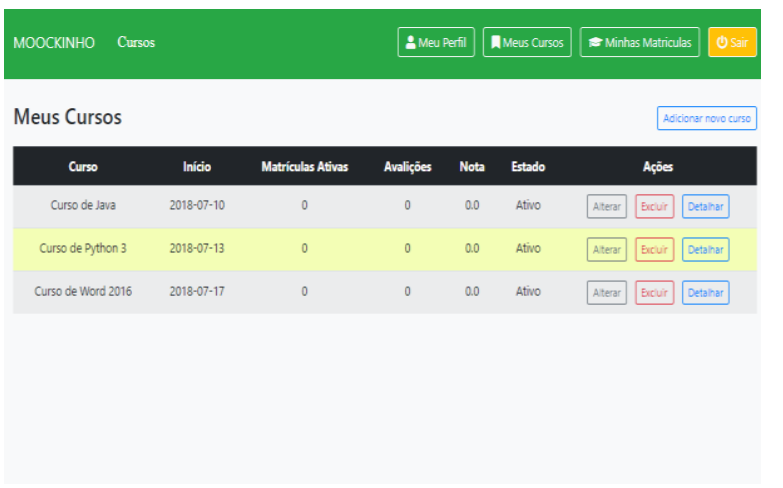

Figura 3a. Gerenciar Cursos

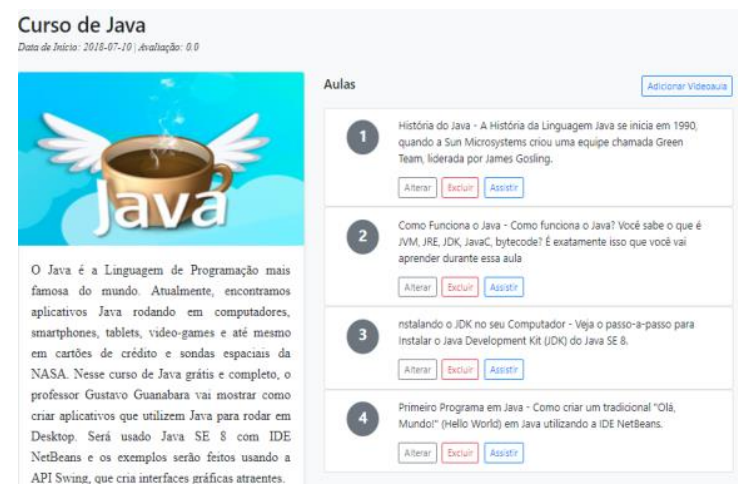

Figura 3b. Gerenciar Videoaulas do Curso

O Moockinho foi desenvolvido e disponibilizado para utilização em $<$ http://lausana.ifrn.edu.br/moockinho $>$. O sistema foi elaborado, projetado, implementado e testado de forma que todas as suas funcionalidades estão passíveis de validação dos seus usuários. Um vídeo demonstrativo do sistema foi disponibilizado em $<$ https://youtu.be/sN0186wMezU>. Através dele é possível verificar uma demonstração das funcionalidades do sistema. Ele é um software livre de código aberto distribuído através da licença creative commons (cc by-sa). Isso quer dizer que qualquer instituição - escolas, universidades, coletivos, indivíduos, organizações privadas ou públicas podem instalar, utilizar, adaptar e criar a partir deste trabalho, mesmo para fins comerciais, desde que the atribuam o devido crédito e que licenciem as novas criações sob termos idênticos).

\section{Considerações finais}

O sistema desenvolvido propõe facilitar a divulgação e gerenciamento de cursos online e, além disso, incentivar a produção e divulgação de cursos online de forma aberta, gratuita e massiva. Por fim, é realizado aqui um levantamento de todas as funcionalidades desenvolvidas e testadas do Moockinho e realizada uma comparação delas com as funcionalidades de outros sistemas de MOOC online (Plataforma TimTec e Plataforma da empresa Curso em Vídeo), conforme apresentado na Tabela 2. 
VII Congresso Brasileiro de Informática na Educação (CBIE 2018)

Anais dos Workshops do VII Congresso Brasileiro de Informática na Educação (WCBIE 2018)

Tabela 2. Comparação dos Sistemas

\begin{tabular}{|c|c|c|c|}
\hline Funcionalidade & Moockinho & TimTec & Curso em Vídeo \\
\hline Listar cursos cadastrados & $\checkmark$ & $\checkmark$ & $\checkmark$ \\
\hline Detalhar curso & $\checkmark$ & $\checkmark$ & $\checkmark$ \\
\hline Realizar cadastro no sistema & $\checkmark$ & $\checkmark$ & $\checkmark$ \\
\hline Realizar login & $\checkmark$ & $\sqrt{ }$ & $\checkmark$ \\
\hline Recuperar Senha & $\checkmark$ & $\checkmark$ & $\checkmark$ \\
\hline Realizar inscrição em curso & $\checkmark$ & $\checkmark$ & $\checkmark$ \\
\hline Gerenciar minhas matrículas & $\checkmark$ & $\checkmark$ & $\checkmark$ \\
\hline Cancelar matricula & $\checkmark$ & $\checkmark$ & $\mathrm{x}$ \\
\hline Assistir videoaula & $\checkmark$ & $\checkmark$ & $\checkmark$ \\
\hline $\begin{array}{l}\text { Visualizar progresso de } \\
\text { aprendizagem }\end{array}$ & $\checkmark$ & $\sqrt{ }$ & $\checkmark$ \\
\hline Gerenciar meu perfil de usuário & $\checkmark$ & $\checkmark$ & $\checkmark$ \\
\hline Solicitar Promoção a administrador & $\checkmark$ & $\mathrm{x}$ & $\mathrm{x}$ \\
\hline Fazer comentários às aulas & $\checkmark$ & $\checkmark$ & $\mathrm{x}$ \\
\hline Avaliar curso & $\checkmark$ & $\mathrm{x}$ & $\checkmark$ \\
\hline Cadastrar cursos & $\checkmark$ & $\mathrm{x}$ & $\mathrm{x}$ \\
\hline Alterar cursos & $\checkmark$ & $\mathrm{x}$ & $\mathrm{x}$ \\
\hline Excluir cursos & $\checkmark$ & $\mathrm{x}$ & $\mathrm{x}$ \\
\hline Cadastrar videoaula & $\checkmark$ & $\mathrm{x}$ & $\mathrm{x}$ \\
\hline Alterar videoaula & $\checkmark$ & $\mathrm{x}$ & $\mathrm{x}$ \\
\hline Remover videoaula & $\checkmark$ & $\mathrm{x}$ & $\mathrm{x}$ \\
\hline
\end{tabular}

\section{Referências}

CREED-DIKEOGU, G. E CLARK, C. Are you MOOC-ing yet? A review for academic.

DA MATTA, Cláudia Eliane; FIGUEIREDO, Ana Paula Silva. MOOC: transformação das práticas de aprendizagem. In: X Congresso Brasileiro de Ensino Superior a Distância. 2013.

INSTITUTO TIM; Portal TIM Tec - MOOC. [2017] Disponível em:

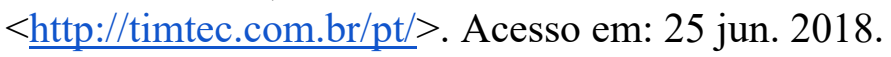

